\title{
The management of Natural Capital. The case of Valencian Country
}

\author{
Josep Lluís Miralles-Garcia ${ }^{1}$
}

1 Universitat Politècnica de València; jlmirall@urb.upv.es

\begin{abstract}
This paper shows the case of Natural Capital management in Valencian Country. The objective is to analyse the efficiency of this management over the last years, and discuss about how we can improve it. Two kinds of aspects have been analysed: some indicators about the evolution of environmental resources; and the urban plans approved by a process of strategic environmental assessment. These analyses allow to know if Valencian Natural Capital improves or not and if the territorial planning processes have been efficient or not. The results allow a discussion about inefficient environmental management and suggest changes to improve it.
\end{abstract}

Keywords: territorial management; strategic environmental assessment efficiency; urban planning and sustainability; Capital Natural

\section{Introduction}

Valencia Country or Valencian Community is a region of Spain with a regional government. In Spain, the regions have the power with legal competence in urban and regional planning. The environmental assessment of plans was regulated by a state Law initially in 2006 through the translation by two laws of European Directive 2001/42/CE about Strategic Environmental Assessment (SEA), and after by a new law in 2013. The Spanish state have power to approve a general or basic environmental rules which must be completed by regional governments, so after initial state regulations, the regional governments began to regulate the environmental assessment of urban and regional plans. In the case of Valencia, this matter is regulated by Law since 2014. Evidently, the European Directive came into force directly about July of 2004 (three years after official publication) while the develop of Spanish and Valencian regulations are developed latter.

On the other hand, in Spain it exists a long experience about urban planning since about 1860 when Ildefonso Cerdà developed the 'Expansion Project of Barcelona'. Progressively, it has been developing a culture of urban planning in society and public administration. This culture includes the role and actions of public administration in order to develop urban plans with a system of public participation and a legal culture according to the sentences of the judicial power. As a consequence of this historical process, when the European Directive was approved, in Spain there was a highly consolidated urban planning system with very specific urban development standards and criteria.

On the contrary, the SEA is a system of public participation on the process of decision taking to approve plans or programmes with consequences on the environment which still is a little experienced system. In fact, the environment is a very transversal reality with many variables that are sometimes related to each other and sometimes not, and in a knowledge framework that is changing continuously, so much of environmental reality is open to interpretation. In addition, the environmental authority to approve the final environmental assessment must be independent of the plan promoter.

In Valencia, these two systems of process for decision-take to planning are being applied in parallel. An only one reality, the territory to be planned, with two parallel systems 
of analysis, planning and decision-take. This situation means that the periods for elabo- 1

rating the urban plans are very long.

And to make matter worse, usually, the times of private investments are short. We can remember, for example, the period of 2-3 years to apply Next Generation funds.

How we can combine all these processes, urban and environmental criteria, public administration efficiency and quickly investment? That is the research question.

The objective of this paper is to show an analyse of the efficiency of urban plan elaborating process in the case of Valencian Country.

\section{Materials and methods}

\subsection{Indicators of Natural Capital evolution in Valencian Country}

Natural Capital includes a list of natural elements, geographically located in a specific territory, which provides natural resources to social and economic activities of this territory or recycles some kind of waste (air pollution, GHG, sewage water ...) produced by the same activities. Therefore, it is possible to generate this list for each particular territory, the territorial area of Valencian Community in our case, and to define indicators as a way to measure the evolution of quantity and quality from that list.

On the other hand, the list of elements of Capital Natural changes over the time, so it is necessary a continuous review of the list to introduce or eliminate elements. This Capital is a kind of capital that can be used in economic activities (together with private, technological, public, human and social capital) according to Ruiz-Tamarit, J.R., Murgui-García, M.J. et Cubel-Montesinos [1] (p. 13) and can explain the economic model of specific territories. That is way some economists study and propose indicators to analyse or characterise the Natural Capital as a base to economic activities [1] (pp. 187-238). But there are many possibilities to identify the Natural Capital that have a significant territorial component [2]. This research is doing a review of the list of natural resources and possible indicators in order to adjust to the goal of its evaluation.

\subsection{Indicators of urban planning management}

Urban plans in Spain are a very powerful regulation. In fact, it exists the criminal punishment in the case of action against the rules, written or graphic, stablished in urban and regional plans focused especially in protected areas. For these reasons, the process to elaborate and approve the plan by public administration is very important. But, on the other hand, the quantity and studies that are made up of urban plans and environmental and urbanistic controls by reports have progressively increased over time.

Regional administration has the competency to approve urban and regional plans. Particularly, Valencian Country has the three departments of Castelló, València and Alacant. Each department has a Territorial Commission to finally approve the urban plans promoted by each municipality. In each case, the technical team of Territorial Commission elaborates the corresponding report with three basic possibilities: approve the plan and finish process; return the plan to municipality to correct; or reject the plan and finish process (usually when municipality don't correct the plan and stopped process). The report includes the historical process to elaborate the plan with the landmark that explain the different phases. The Valencian Community has 542 municipalities and the department of València has 266 municipalities. Currently, all these municipalities have their own plan elaborated in different historical time according to the Urban Act valid in each moment.

In this situation it is possible to analyze the process of elaborate urban plans according to information of corresponding reports and is possible to stablish indicators such a period of time to finish process, number of times it has been a public participation process, or others.

The research is focused on the period from 2015 October to 2021 October for all municipalities of department of València. The number of municipalities of València is $49.08 \%$ 
of all municipalities of Valencian Community, so it is a significant sample of real situation. 1 The period is significant of current situation.

\section{Results about efficiency of environmental processing}

It's known that there are many studies about environmental indicators, so this section is focused to the other goal, that is, about efficiency of environmental processing.

Regional administration of Valencia has two ways to supervise the process of elaboration and approval urban plans: environmental and urbanistic supervision. These two ways are monitored by two different public organisms: the Assessment Environmental Commission (CEA) and the Urban Territorial Commission (CTU). In addition, there are three delegations of CTU, one for each department of Castelló, València (CTUV) and Alacant.

Basically, the municipalities by City Council promote the elaboration of urban plans including a process of public participation on decision-make. To do this process, initially City Council elaborates a first version of plan and environmental assessment that also send to different public and private organisms to inform it (about 20-30). After public participation, City Council elaborates a second version of urban plan and approve it. This second version will send to both CEA and CTU (CTUV in the case of department of Valencia). CEA elaborates an environmental assessment with conditions and corrections that are mandatory. Usually, this report has many conditions about any environmental issue according to laws or the own criteria and it may take about two years to issue. On the other hand, CTU issues a report according to the reports sent by the different public and private organisms, the environmental assessment of CEA and checking urban technical characteristics according laws. The report can conclude in different ways: urban plan finally approved, partial approved (approved all its content except some very specific aspect to correct), delayed to corrections (in process), rejected (usually when process in not correct and it is necessary restart it) and retirement (when municipalities decide abandon the process).

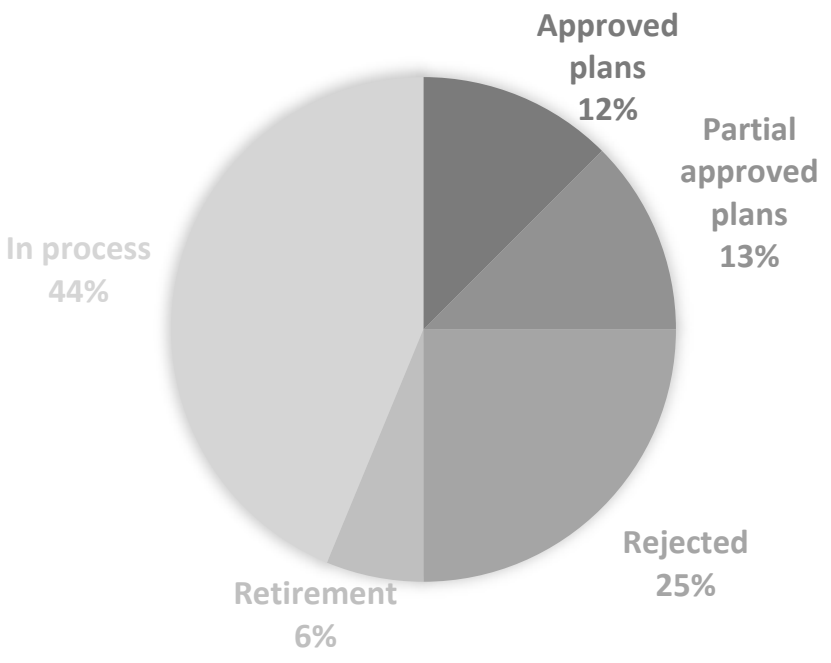

Figure 1. Management results of CTUV (Valencia, Spain) between 2015-2021 about urban planning.

Figure 1 shows the results of urban plans managed by CTUV in the period 2015-2021. The total of urban plans reported was 32 . Between them, only 4 was finally approved. The majority (14) are in process.

Table 1 shows indicators about the four urban plans approved in the period 20152021. Note you that the department of Valencia has 266 municipalities and, as a general criterion, the urban plans are designed for ten years. In consequence, the frequency of urban plan renovation should be about 27 municipalities by year. This indicator evidences 
an anormal situation. In fact, as now we will see, the process to elaborate urban plans is 1 complicated and very expensive in time, therefore, also economically. On the other hand, 2 currently all municipalities of Valencian Country have an urban plan in force, so can main- 3 tain urban activity and, in general, no necessarily need to renovate it urban plan.

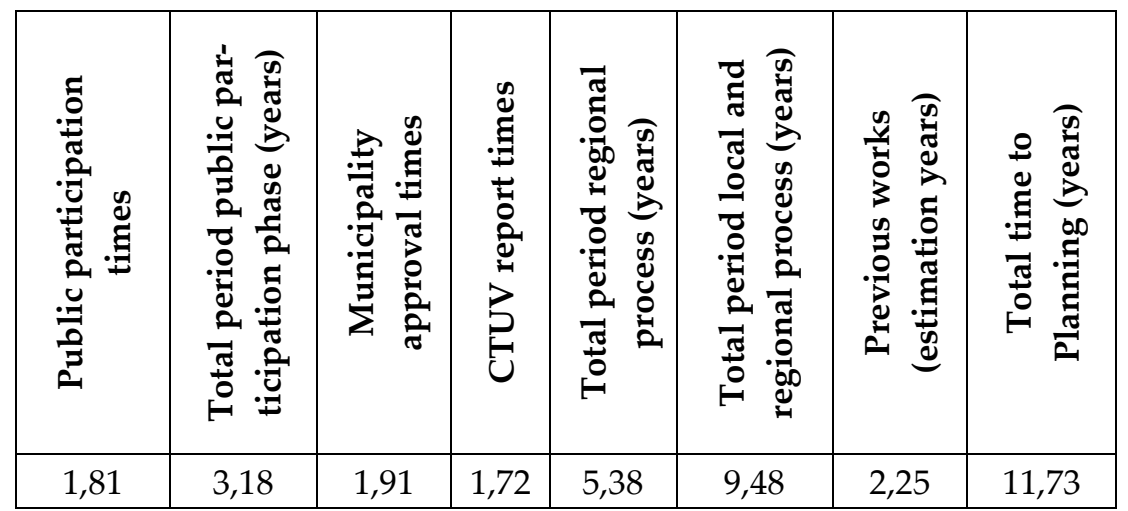

Table 1. Indicators of mangement about urban plans approved by CTUV, 2015-2021.

As you can see in Table 1, the average time to complete the process to renovate the urban plan is 11,73 years, that is, more than the horizon time to design the plan. The average of number of times that the plans had public exposition is about 2, the average of total period of public participation phase was about 3 years and the average of total period of regional revision by the CTUV was more than 5 years because of the successive corrections. In addition, the environmental assessment is valid for 3 years so, if the review phase of plan makes more than 3 years, it is necessary to restart the environmental assessment process with probably new conditions, which greatly delay the approval process.

On the other hand, a not rigorous process of approval process generates legal uncertainty with important consequences and difficulties [3].

\section{Conclusion}

It is necessary to review the process of approval urban plans which are an essential tool to manage the Capital Natural and natural resources. Some authors are studying how is possible to rethink the system and recover urban planning as a significant tool to manage efficiently the territory [4]. No planning is not an alternative in a globalized world where human activities have a greatly destruction capacity. The destruction of territory is practically irreversible. But solutions by planning can not waiting for years and years. It is necessary to find the equilibrium point.

Funding: This research received no external funding.

Acknowledgments: I want acknowledge the technicians of Urban Territorial Commission of Valencia for they hard work in order to rationalize the process of approval urban plans helping the municipalities.

Conflicts of Interest: The authors declare no conflict of interest

7

8

9

10

11

12 